\section{A case report of bilateral uveitis with meningococcal septicaemia resulting to blindness and adjustment disorder}

\author{
Zaharaddeen Garba Habib, ${ }^{1}$ \\ Saudat Garba Habib, ${ }^{2}$ Safiya Gambo, ${ }^{3}$ \\ Sadiq Hassan, ${ }^{2}$ Jamila Sani $^{3}$ \\ ${ }^{1}$ Department of Psychiatry, Aminu Kano \\ Teaching Hospital and Bayero \\ University Kano; ${ }^{2}$ Department of \\ Ophthalmology, Aminu Kano Teaching \\ Hospital and Bayero University Kano; \\ ${ }^{3}$ Department of Paediatrics, Murtala \\ Muhammad Specialist Hospital, Kano, \\ Nigeria
}

\begin{abstract}
To report the rare case of a patient with bilateral uveitis with meningococcal septicaemia from Neisseria meningitides, resulting to blindness and adjustment disorder. A 9-years-old boy presented to the Emergency paediatric unit of a hospital with complaints of fever, and neck stiffness of one week duration. There was associated sudden decreased vision, pain and photophobia of both eyes. Neck was stiff and retracted with positive Kernig's and Brudzinski's signs. Neisseria meningitidis was isolated from both the turbid CSF and the blood. Visual Acuity (VA) was Perception of Light (PL) and Counting Finger (CF) in right and left eyes (RE and LE) respectively, with 360 degrees posterior synechiae and pupillary membrane. Diagnosis of meningococcal septicaemia with bilateral uveitis was made. Patient was treated with IV ceftriaxone, sub-conjunctival dexamethasone, dexamethasone, atropine and moxifloxacin eye drops and other supporting medications. Although he showed improvement of VA to RE-6/36, LE- 6/24, he absconded from follow-up and later relapsed with subsequent blindness (VA of Perception of Light in Both eyes) and symptoms of adjustment disorder. Although ocular involvement is rare in meningococcal septicaemia, patients should always have detailed ophthalmological examination as prompt diagnosis and management could improve the visual outcome.
\end{abstract}

\section{Introduction}

Meningococcal meningitis is a disease caused by Neisseria meningitides. One of the commonly reported epidemics within the meningitis belt of the African sub region was that of 2009 attributable to Neisseria meningitides sero group A. Northern Nigeria and Niger accounted for about $85 \%$ of cases. ${ }^{1,2}$ This was followed by mass vaccination with menafric. ${ }^{3-5}$

Children with Nesseria meningitides meningitis could develop complications such as purpura fulminans, polyarthritis and cutaneous vasculitis. ${ }^{2}$ Complication such as bilateral uveitis and endophthalmitis are rare. ${ }^{6}$

Even though this intraocular inflammation is rare it may be caused by spread of the micro-organisms from the primary site of infection haematogenously penetrating the blood ocular barrier to the eye (secondary site). ${ }^{7}$ Factors such as immune suppression, prolonged Intensive care unit stay and intravenous drug use have been reported to be some of the predisposing factors. ${ }^{8}$ Blindness could result from meningitis through raised intracranial pressure leading to papilloedema or disc oedema, it could also be as a result of infiltration of the optic nerve with resulting neuropathy. It could rarely be as a result of cortical blindness, sterile reactive uveitis or endophthalmitis. Adjustment disorder is the development of emotional or behavioural symptoms in response to an identifiable stressor(s) occurring within 3 months of onset of the stressor. ${ }^{9}$ We are reporting this case to highlight that default from follow-up and management could lead to relapse and blindness in uveitis associated with meningitis.

\section{Case Report}

We present a 9-years-old primary 3 boy, who presented to the EPU of a tertiary health facility with complaints of fever and neck stiffness of 1 week duration. The fever was high grade, continuous, briefly relieved by analgesics. There was sudden decreased vision and photophobia in both eyes with associated headache but not convulsion, loss of consciousness or hearing loss.

He is the $8^{\text {th }}$ child in a monogamous setting of 10 children. His father died from a short illness 2 weeks prior to presentation; the mother is a petty trader. The patient had a delayed grief reaction. Since father's death the family has found living difficult. They earn 250 to 300 naira per day (about 0.5 to 0.75 USD).

Physical examination revealed a child with altered sensorium (Glasgow coma score - 11/15), febrile (temperature of $40^{\circ} \mathrm{C}$ ), weight and height of $17 \mathrm{~kg}$ and $117 \mathrm{~cm}$. His neck was stiff with positive Kerning's and
Correspondence: Saudat Garba Habib, Department of Ophthalmology, Aminu Kano Teaching Hospital and Bayero University Kano, Kano, Nigeria

E-mail: garbasaudat7@gmail.com

Key words: Meningococcal septicaemia; uveitis; blindness; adjustment disorder

Conflict of interest: The authors have no conflict of interest to declare.

Availability of Data and Materials: All data underlying the findings are fully available.

Ethics approval and consent to participate: No ethical committee approval was required for this case report by the Department, because this article does not contain any studies with human participants or animals. Informed consent was obtained from the patient included in this study.

Consent for publication: The patient's parent gave her written consent to use the child's personal data for the publication of this case report and any accompanying images.

Received for publication: 7 July 2020.

Revision received: 13 September 2020.

Accepted for publication: 4 February 2021

This work is licensed under a Creative Commons Attribution NonCommercial 4.0 License (CC BY-NC 4.0).

${ }^{(C)}$ Copyright: the Author(s), 2020

Licensee PAGEPress, Italy

Pyramid Journal of Medicine 2020; 3:81

doi:10.4081/pjm.2020.81

Bruzunski's signs. Pulse rate was 98 bpm, regular, moderate volume with normal heart sounds. His respiratory rate was 35 breaths/min, and had $\mathrm{SpO} 2$ of $90 \%$. Ophthalmological findings include Visual Acuity (VA) unaided: Perception of Light (PL) - Right eye and Counting fingers (CF) at $1 \mathrm{~m}-$ Left eye. We couldn't assess color and contrast sensitivity because of poor vision. There was mucopurulent discharge from both eyelids, conjunctivae were hyperaemic, with grade 2 corneal haziness and centrally located keratic precipitates, fresh and pigmented. There was flare and cells but no hypopyon in the anterior chamber. Pupil had a 360 degree posterior synechiae with fibrinoid pupillary membrane which obscured further view in both eyes.

Intraocular pressure measured with applanation tonometer was $12 \mathrm{mmHg}$ and $11 \mathrm{mmHg}$ in the right eye and left eye respectively. There were no significant findings in other systems. 


\section{Investigations}

i) Ocular B-Scan done showed clear lens with vitreous opacities.

ii) Vitreous tap was done however, microscopy culture and sensitivity of the vitreous sample were negative. Eye swab for $\mathrm{m} / \mathrm{c} / \mathrm{s}$ yielded no growth.

iii) Lumbar puncture revealed turbid Cerebrospinal Fluid (CSF) with raised opening pressure. The CSF had elevated protein $(0.6 \mathrm{~g} / \mathrm{l})$ and low glucose $(20 \mathrm{mg} / \mathrm{dL})$ content.

Random blood glucose was $7.4 \mathrm{mmol} / \mathrm{L}$. Full blood count showed leucocytosis of $13.4 \times 10^{9}$ with neutrophilia. Both CSF and blood cultures isolated Neisseria meningitidis serotype $\mathrm{C}$, sensitive to Ampicillin, Ceftriaxone, Chloramphenicol, Ciprofloxacin and Meropenem. He had hypokalemia, other electrolytes were normal. The diagnosis of Meningococcal meningitis with bilateral uveitis was made, the patient was given intravenous Ceftriaxone (100mg/kg/day), Dexamethasone $(0.15 \mathrm{mg} / \mathrm{kg} 6$ hourly) for 2 days, Paracetamol, oxygen supplementation and intravenous fluids. Ocular treatment included sub-conjunctival dexamethasone $2 \mathrm{mg}$ daily for 3 days, dexamethasone eye drop hourly for 48 hours, then 2 hourly for 12 days, then 4 hourly thereafter. Atropine eye drop 8 hourly and MOXIFLOXACIN eye drops 4 hourly were also given.

After 3 weeks on admission, the child improved. Visual acuity unaided RE- 6/36, LE- 6/24.Corneal haziness had reduced, lens was clear and the pupillary membrane partially cleared with a visible faint fundal red reflex. He was discharged on dexamethasone, atropine and moxifloxacin eye drops. He was lost to follow up until two months later when he presented with recurrence of ocular symptoms and signs, visual acuity of PL in both eyes and has not improved since then.

At about 13 months after his initial admission, he developed behavioural problems. The neuropsychological assessment found that patient has visual impairment; there was no impairment in other sense organs. He had no focal neurological signs and no thought, perceptual disturbances or cognitive deficit. Psychometric assessment with the Cambridge Inventory Neurological, Reporting Questionnaire for children and the Centre for Epidemiological Studies Depression scale for Children (CESD-C) indicated patient was experiencing an adjustment reaction with an anxiety component. The anxiety becomes more pronounced whenever he goes to school.

At present, patient has received counselling for the adjustment disorder and has been enrolled into the school for the blind so that he can learn braille and vocational skill.

\section{Discussion}

Ocular involvement in patients with Meningococcal meningitis has been discussed by Shuttle et al. ${ }^{10}$ This is in keeping with the protean manifestations of the disease. Our patient was found to have bilateral uveitis similar to what was reported by Faye et al. ${ }^{11}$ They observed eye involvement in one of the 5 paediatric patients admitted with meningococcal infection over a 2 and a half-year period in France. ${ }^{11}$ However, the patients they described had the W135 subtype while ours had the $\mathrm{C}$ type meningococcal disease. These differences in subtype can be accounted for by regional predilection. The $\mathrm{C}$ subtype now predominates the A type in Western Africa ${ }^{12}$ while other strains like the W135 is commoner in Europe. ${ }^{12}$ Involvement is usually bilateral but unilateral involvement is not uncommon and has been documented. ${ }^{10}$

In the case presented here the patient had bilateral uveitis that we think may be a sterile reactive uveitis. He initially showed improvement of ocular symptoms, the patient defaulted and when he came back, he had seclusion pupillae with a membrane and posterior synechiae obscuring further view of the posterior segment where he may have posterior uveitis.

This was confounded by delayed presentation and lack of adherence to treatment which can be explained by the poor financial status of family. The patient's maladjustment stemmed from educational difficulty coupled with lack of vocational training and difficulty with mobility. This is in line with findings from Ilorin, Nigeria. ${ }^{13}$

It is to address these issues that patient was enrolled at the school of the blind. This will help him with educational and vocational learning. The process of adjustment to blindness is a process that takes time as one goes through the phases of trauma, shock and denial, mourning and withdrawal, succumbing and depression, reassessment and reaffirmation, coping and mobilization and self-acceptance and self-esteem as elucidated by Tuttle and Tuttle. ${ }^{14}$ The patient seems to be in stage four and will likely reach stage seven quickly based on the management instituted.

\section{Conclusions}

The presentation of meningococcal meningitis is protean, associated ocular morbidity are not uncommon. Prompt diagnosis, intervention and adherence to medications are associated with good prognosis.

\section{References}

1. World Health Organisation Multi Disease Surveillance Center (MDSC), WHO/African region. Meningitis Weekly Bulletin.

2. Iliyasu G, Lawal H, Habib AG, Hassan-Hanga F, Abubakar I S, Bashir $\mathrm{U}$, et al. Response to the Meningococcal Meningitis Epidemic (MME) at Aminu Kano Teaching Hospital, Kano (20082009). Nig J Med 2009;4:428-30.

3. WHO/PATH Meningitis Vaccine Project. Press release: dramatic fall in cases of meningitis A in three West African nations after new vaccine introduction. Health Aff 2011;30:1049-57.

4. Kristiansen PA, Diomandé F, Ba AK, Kristiansen PA, Diomandé F, Ba AK, Sanou I, Ouédraogo AS, Ouédraogo R, et al. Impact of the serogroup A meningococcal conjugate vaccine, MenAfriVac, on carriage and herd immunity. Clin Infect Dis 2013;56:35463.

5. Daugla DM, Gami JP, Gamougam K, Naibei N, Mbainadji L, Narbé M et al. Effect of a serogroup A meningococcal conjugate vaccine (PsA-TT) on serogroup A meningococcal meningitis and carriage in Chad: a community trial. Lancet 2013;383:40-7.

6. Shah V, Garg A, Stanford M. Endogenous Meningococcal Endophthalmitis with Isolated Joint Involvement in Immunocompetent Adults. Ocular Immunol and Inflamm 2018;26:523-6.

7. Balaskas K, Potamitou D. Endogenous endophthalmitis secondary to bacterial meningitis from Neisseria Meningitidis: a case report and review of the literature. Cases J 2009;2:149.

8. Ness T. Endogenous endophthalmitis. Ophthalmologe 2007;104:935-9.

9. ICD 11- Mortality and Morbidity Statistics. Retrieved on 2019-08-28. Available from: http://id.who.int/icd/entity/334423054

10. Shuttleworth FN, Benstead JG. Primary Meningococcal Ophthalmia. BMJ 1947;2:568-9.

11. Faye A, Mariani-Kurkjian P, Taha MK, Louzeau C, Bingen E, Bourrillon A. et al. Clinical aspects and outcome of meningo-coccal disease due to Neisseria meningitides of serogroupW135 in 5 children. Archives de pédiatrie: Organe official delaSociétéfrançaise de pédiatrie. Arch Pediatr 2005;12:291-4.

12. Mohammad I, Garba I, Abdulrazaq GH. Emergence and control of epidermic meningococcal meningitis in 
Subsaharan Africa. Pathog Glob Health 2017;111:1-6.

13. Tunde-Ayinmode MF, Akande TM, Ademola-Popoola DS. Psychological and Social adjustment to blindness:
Understanding from two groups of blind people in Ilorin, Nigeria. Ann Afr Med 2011;10:155-64.

14. Tuttle D, Tuttle NR. Self-esteem and Adjusting with blindness: The process of responding to Life's demands. 3rd Edition. Spring field, IL: Charles C. Thomas; 2004. 